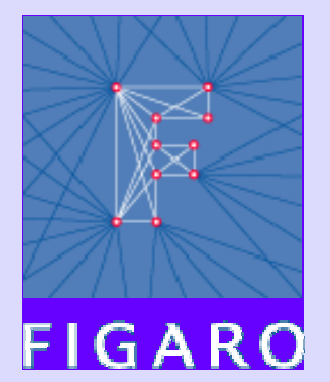

\title{
FIGARO and Open Access to Electronic Information Objects
}

Presentation of the initiative and of some related technical considerations

Dr. Stefan Gradmann

Regionales Rechenzentrum der Universittät Hamburg

stefan.gradmann@irzunj-hamburg.de 


\section{Overview}

FIGARO

... The FIGARO project

ㄱ.? Objectives and Partners

[.. Open Access in FIGARO/ GAP

ㄱ.. Organisaitional, economic, legal, policy aspects of -Open Access" mostly have been covered by Bas Savenije in his talk in Louvain (Oct. 2002)

ㄱ. Technologjical aspects are the core of this presentation

- Open, vendor indlependlent document modlels

- Poining, linking, identifying

- Authentication, authorization 


\section{Why FIGARO (and GAP)?}

FIGARO

... The critical situation in scholarly publication and communication forces universities to act in their role as content generators and users of content (much could be said about the schizoid position of scientists in the line of Stevan Harnad in that respect ...)

... The internet is evolving into the primary publication and communication platform in an increasing number of disciplines

... Digital publication still is heavily modeled on the print-analogy: the innovative potential of electronic platforms is almost not used at all.

... Individual university presses are too weak (economically and technically speaking) to change these basic contextual parameters

"German Academic Publishers" (GAP, funded by DFG, kicked off 01.12.2001) and FIGARO (funded by EC, kicked off 01.05.2002) to create a technical and organizational co-operation model for academic e-publishers. 


\section{FIGARO: Objectives}

FIGARO

[.. Overall: stimulate and support scientific communication and return science to scientists by

... Building an open, Europe-wide co-operation framework for federating academic e-publishing institutions including

$\square$ Shared/distributed technical facilities, e. g.

- Shared WWW-based workflow

- Supporting tools for open, standard based object modeling

- Generic authentication layer pluggable in SSO architectures

‥ Common organisational/ exploitation components, e. g.

- Business model

- Legal framework

[.. Make this framework sustainable

... Investigate new models of article publishing ('post-journals') and of quality assurance ('public peer reviewing) 


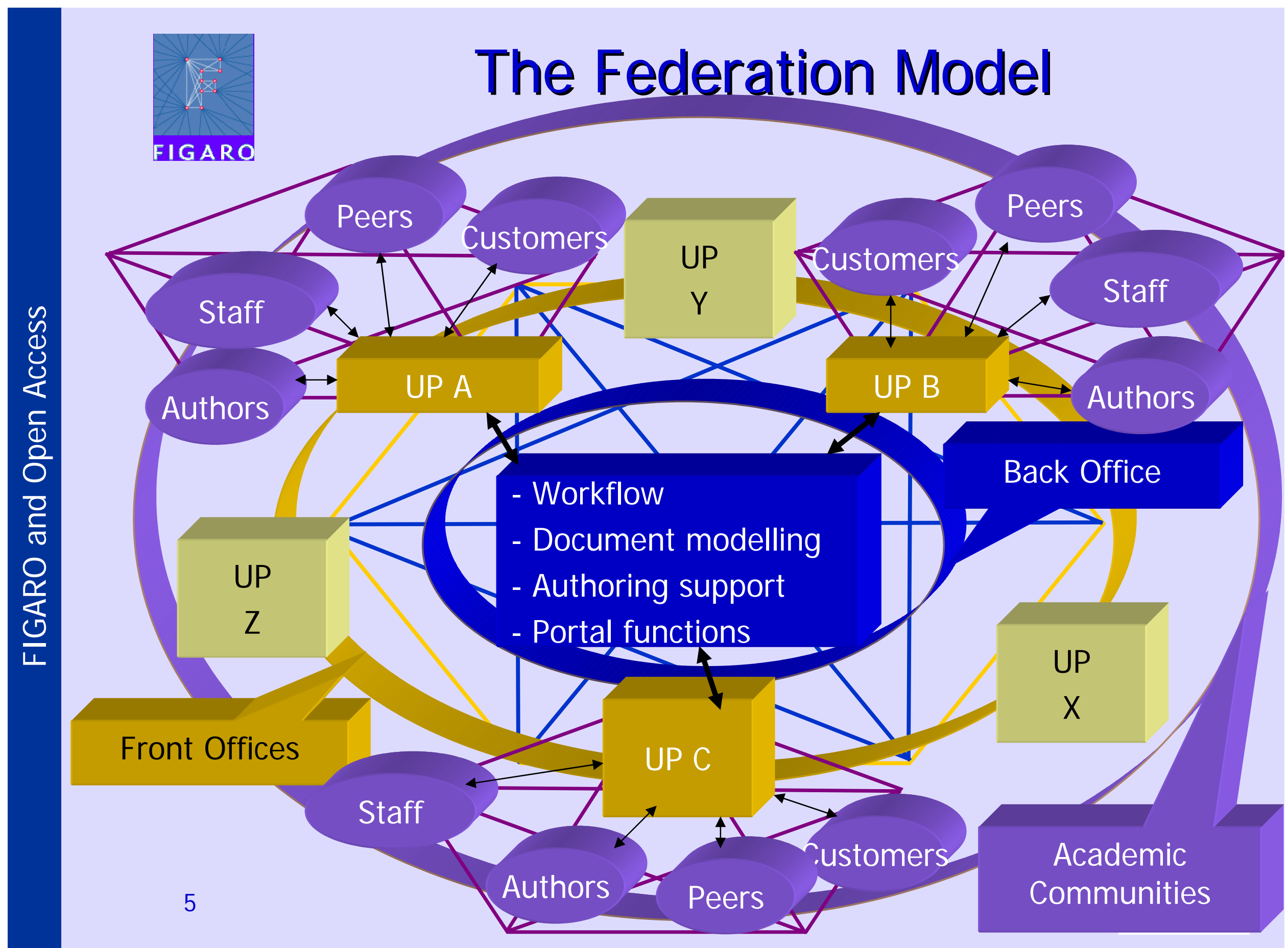




\section{The FIGARO Consortium}

FIGARO

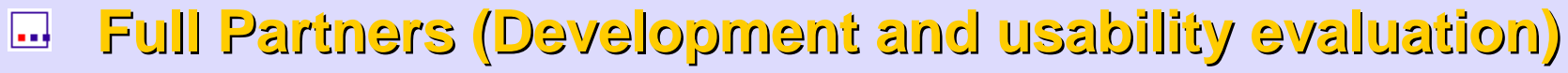

$\square$ Utrecht University (Consortium Leader) and Delft University $(\mathrm{NL})$

Lamburg University (Technical Coordination) and Oldenburg University (D)

Daidalos bv IT in Publishing (NL)

$\square$ Firenze University (I)

... Assocjalce Psirisers (Coriterit Provjsjors)

$\square$ Adademic content providers: Stichting Delft Cluster (NL), Leuven University (B), Lund University (S)

SME publishers: Uitgeverij LEMMA B.V. (NL) and Wydawnictwo DiG SC. (PL)

Association of Research Libraries/SPARC (US)

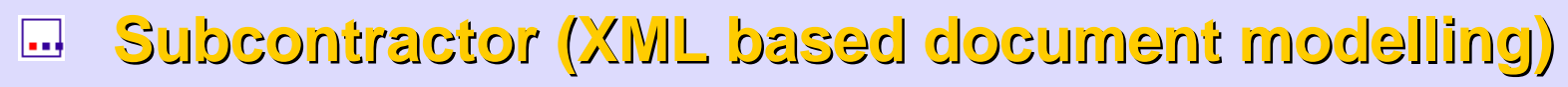

SUN Microsystems/StarOffice (D) 


\section{Standard Based Innovation as a basis for Open Access \\ FIGARO}

... Achieve functional innovation via integration and adaption of standard based (and wherever possible open source) building blocks and do not start own developments we cannot sustain

.. Examples of such standards:

ㄱ. Metadata (has been covered by Andy and often is overestimated, anyway)

ㄱ. $\mathrm{OAl}$-protocol (covered by $\mathrm{Andy}$, as well)

? I Open, generic document models expressed in XML (Schema) and derived from operational modeling proposals such as DocBook and OO-XML

ㄱ. Open, URN-based linking and pointing

ㄱ.. Open, generic authentication methods using LDAP 


\section{Functional Building Blocks}

FIGARO

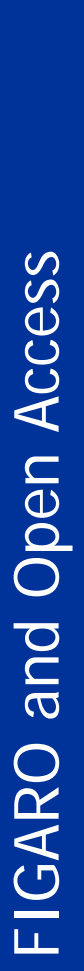

XML based De r ume nt Management an : oui p (O) (XML to poif / h. ml.

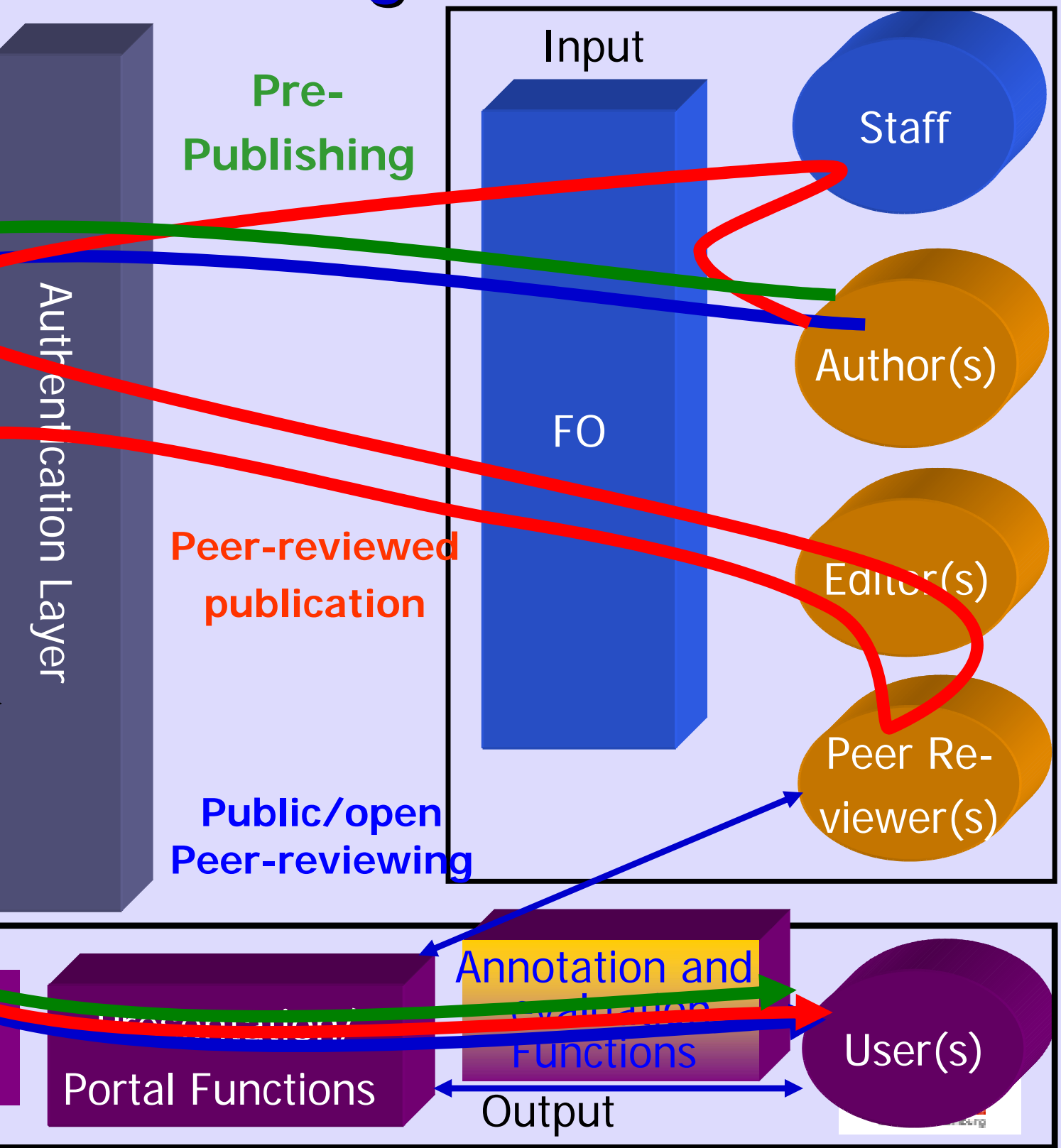




\section{Document Modeling}

\section{FIGARO}

.. Use standard based, open models for digital information objects in authoring support and to support new and innovative publication objects ,electrified" publishing

$$
\text { „real" e-publishing }
$$

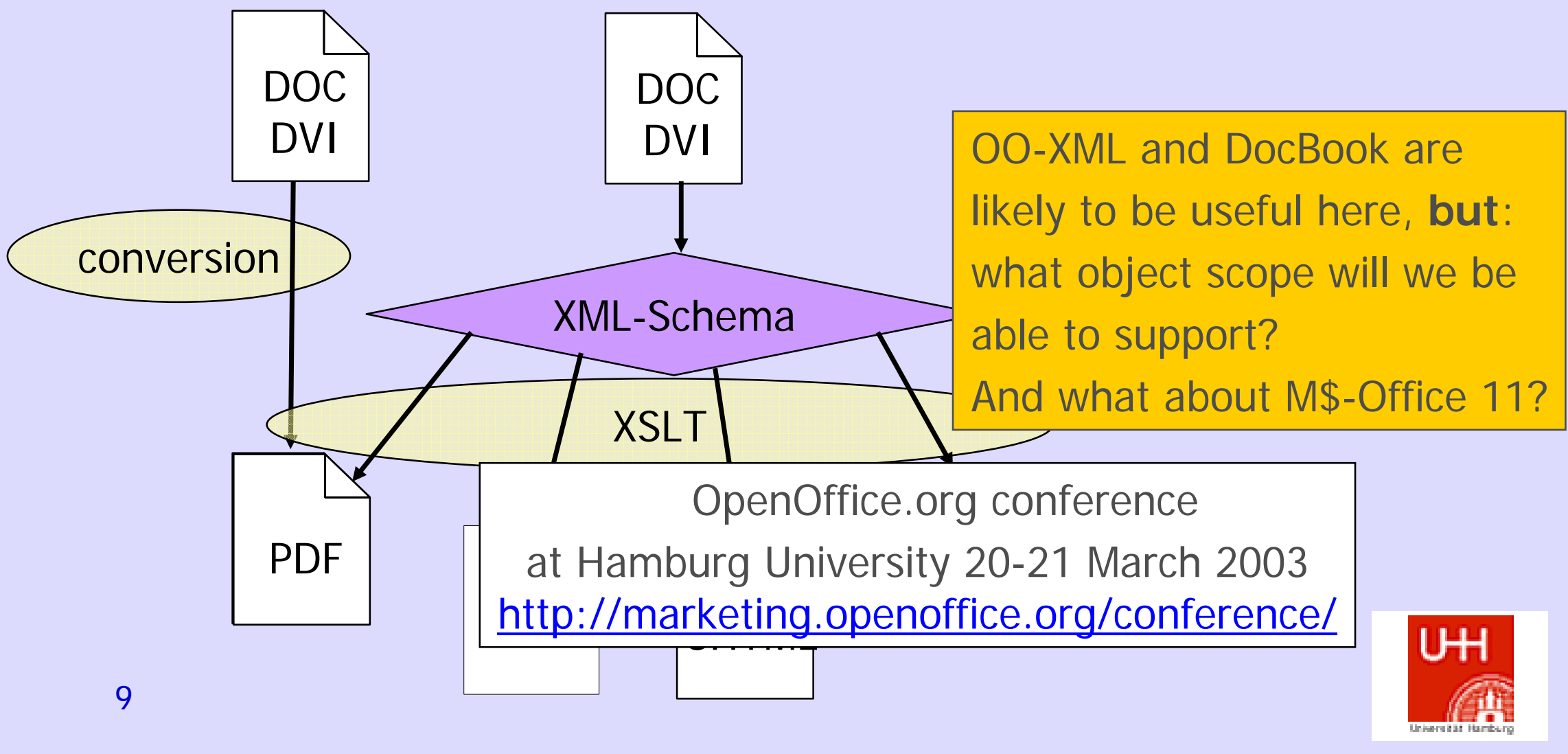




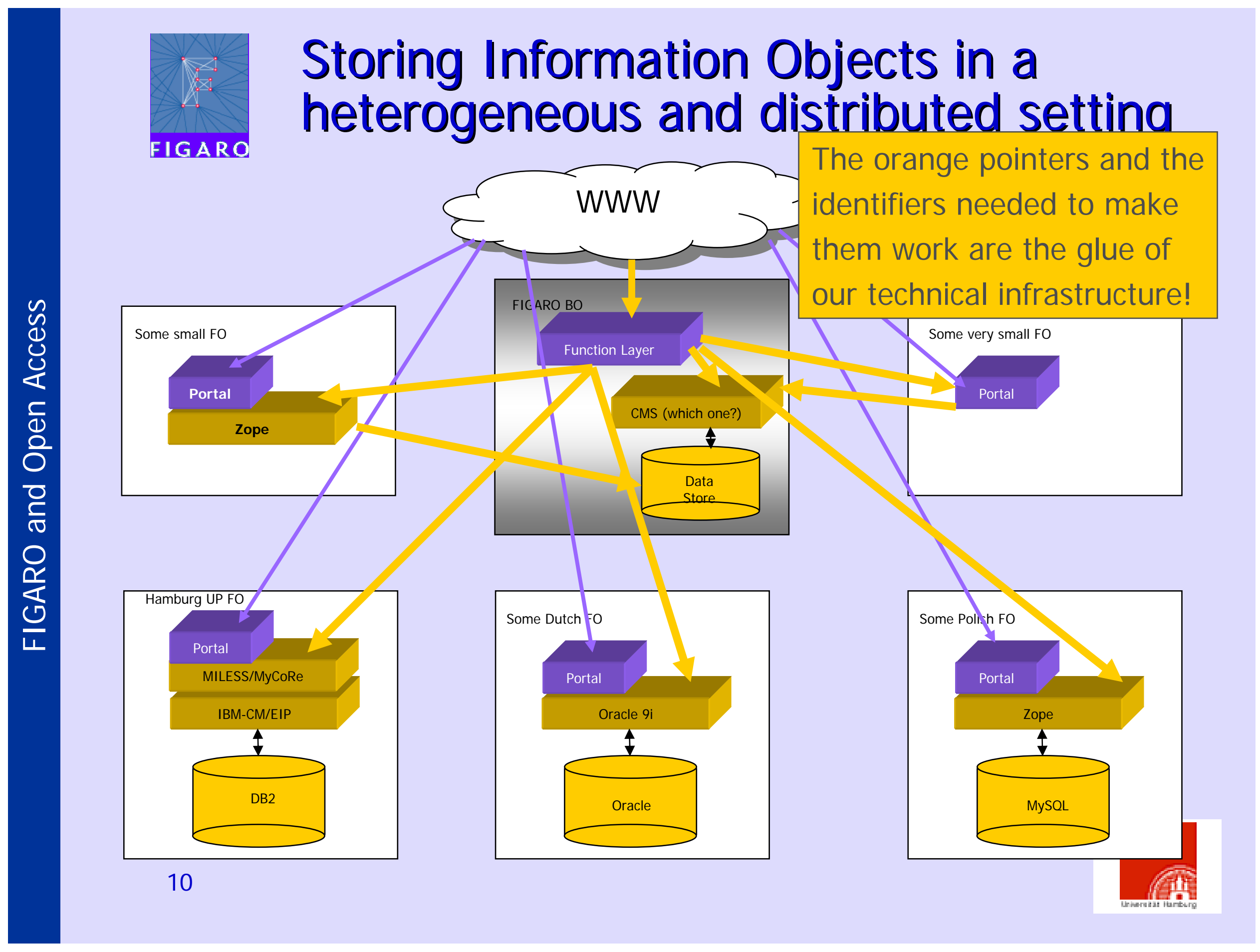




\section{Open Access and Pointers \& I dentifiers: some lessons learned}

[... Full grown CMSs are degraded to simple digital object stores in such an approach

[.. Details regarding pointers and identifiers

I. URL will not do the job (mind persistency aspects and the longevity of scholarly quotations!)

… XLink \& related standards are intensely observed, but not yet a sure bet

.. We may well go for URN - but then have to determine a syntax, find resolving partners etc.

ㄱ. And: beware of DOI ...! 


\section{Open Access and Authentication FIGARO \& Authorization}

... $\bigvee / H O$ - e. g. authors, customers, editors, reviewers, annotators ...

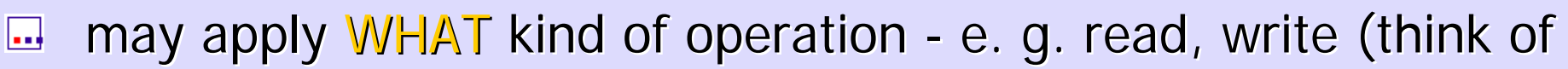
collaborative authoring!), annotate, stabilize ("freeze"), apply different status-levels such as 'rejected' 'ready for public reviewing', copy/attempt pirating

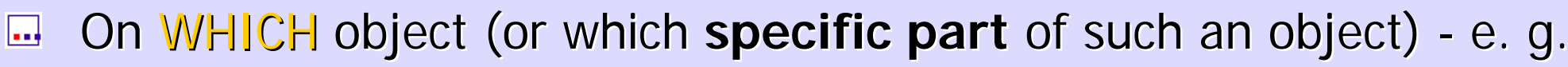
overall document ID but also micro-structures to be referenced as part of compound MM-documents as well as of uniform complex objects ('books' and the like)

... In which CONГГE $\nearrow Г$ - e. g. "scientific use" (teaching/studying) vs. commercial use, pre-publishing, public reviewing, publishing etc.

... In other words: identify Actors, Enjiticjes, Operations, Conitext and organize these in a 4-climensional rnatrix in a secure, reliable way using available building blocks and starnclarcls wherever possible 


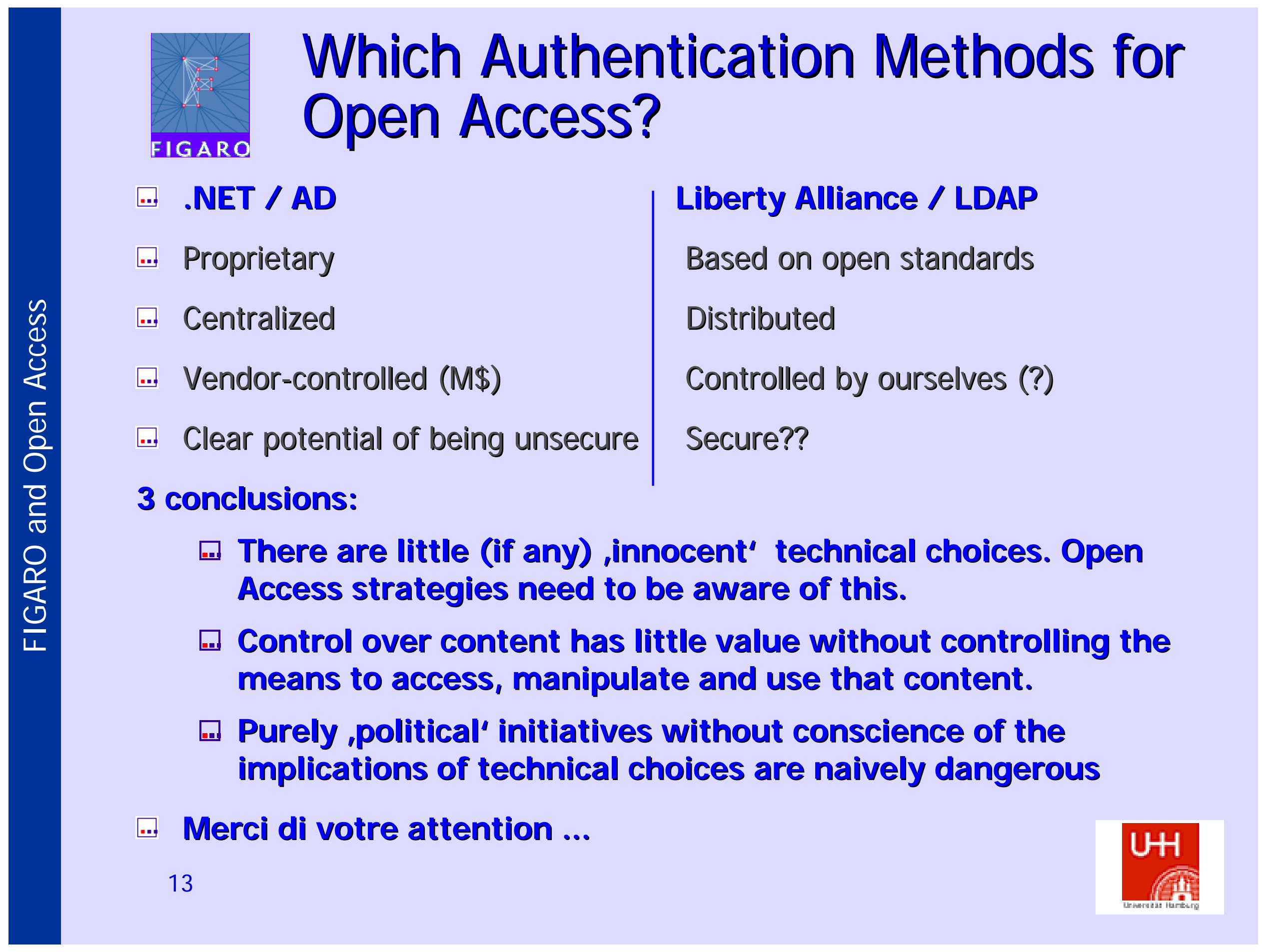

